\title{
P3071
}

\section{Manganese does not potentiate the neurotoxicity of MPTP}

\author{
金 良昊 ${ }^{1}$ 、Baek Sun Yong ${ }^{2}$ Kim Young Hoon ${ }^{3}$ 、朴 正鮮 ${ }^{4}$ \\ ${ }^{1}$ Department of Occupational and Environmental Medicine Ulsan University Hospital、 \\ ${ }^{2}$ Busan University Hospital、 ${ }^{3}$ Medical College, Inje University、 ${ }^{4}$ 韓国産業安全公団
}

The importance of environmental agents such as manganese $(\mathrm{Mn})$ and pesticides in the etiology of Parkinson's disease (PD) is gaining recognition. However, data on the role of $\mathrm{Mn}$ as a risk factor for $\mathrm{PD}$ is contradictory. Animal model data on the contribution of $\mathrm{Mn}$ to the development of parkinsonism is also contradictory. We utilized a mice model of parkinsonism to: (1) evaluate 1-methyl-4-phenyl-1,2,3,6-tetrahydropyridine (MPTP)-induced neurotoxicity; (2) evaluate whether Mn exposure can affect MPTP-induced neurotoxicity.

The present study utilized a MPTP mice model of parkinsonism and a $2 \times 3$ experimental design (MPTP $\times \pm \mathrm{Mn}$ ) as follows,: SS; MPTP(-) $\times$ Mn(-); SLMn, MPTP(-) $\times$ low Mn(+); SHMn, MPTP(-) $\times$ high Mn(+); MpS: $\operatorname{MPTP}(+) \times \mathrm{Mn}(-) ; \mathrm{MpLMn}, \operatorname{MPTP}(+) \times$ low $\mathrm{Mn}(+) ; \operatorname{MpHMn}, \mathrm{MPTP}(+) \times$ high $\mathrm{Mn}(+)$ (Each group contained seven to ten mice.)

Male C57BL/6 mice ( 8 weeks old, body weight $25-30 \mathrm{~g}$ ) were caged in an air-conditioned room maintained at $22 \pm 2{ }^{\circ} \mathrm{C}$, relative humidity $50 \pm 10 \%$, with a $12 / 12 \mathrm{~h}$ light/dark cycle. We administered MPTP to MpS, MpLMn, and $\mathrm{MpHMn}$ group via an intraperitoneal injection of $20 \mathrm{mg} / \mathrm{kg} /$ day four times every two hours. The SS, SLMn, and SHMn group received the same volume of saline intraperitoneally. After that, MpLMn and SLMn group were treated intraperitoneally with $0.2 \mathrm{ml}$ of $\mathrm{MnCl}_{2} 4 \mathrm{H}_{2} \mathrm{O}$ (2mg/kg body weight), and the $\mathrm{MpHMn}$ and SHMn group were treated with $0.8 \mathrm{ml}$ of $\mathrm{MnCl}_{2} 4 \mathrm{H}_{2} \mathrm{O}$ (8mg/kg body weight) once a day for 3 weeks. The SS group received the same volume of saline intraperitoneally. After the last $\mathrm{Mn}$ injection, the mice were sacrificed.

Blood $\mathrm{Mn}$ levels were elevated in the Mn-exposed groups. The number of tyrosine hydroxylase (TH)-immunoreactive (ir) neurons in the substantia nigra pars compacta ( $\mathrm{SNpc}$ ) were decreased significantly in the MPTP-exposed groups (MpS, MpLMn and MpHMn ) in comparison with non-exposed groups (SS or SLMn, SHMn). The densities of TH- ir axon terminals in caudate-putamen (CPU) were significantly decreased in MPTP-treated groups, compatible with a decrease of TH-ir neurons in the SNp. However, Mn treatment did not affect the densities of TH-ir axon terminals in the CPU nor the number of TH-ir neurons in the SNpc.

MPTP treatment resulted in a reactive astrocytosis, which was shown as a profound increase in GFAP immunoreactivity in the CPU or globus pallidus (GP). The densities of GFAP-ir astrocytes in the CPU or GP were significantly increased in the MPTP-treated groups in comparison with MPTP non-treated groups. But, Mn treatment did not affect MPTP neurotoxicity.

Concentrations of dopamine (DA) in the corpus striatum were decreased significantly in the MPTP-exposed groups only, but $\mathrm{Mn}$ had no effect. DA reduction was $85-90 \%$. Concentrations of DA metabolites such as 3,4-dihydrophenylacetic acid (DOPAC) and homovanillic acid (HVA) in the corpus striatum were also decreased significantly in MPTP-exposed groups only, showing a similar pattern to those of DA (the reduction was approximately $60 \%$.). Thus, decreased dopaminergic activities with MPTP led to decreased DA and its metabolites. In summary, MPTP targeted dopaminergic systems whereas Mn does not potentiate the neurotixicity of MPTP. 